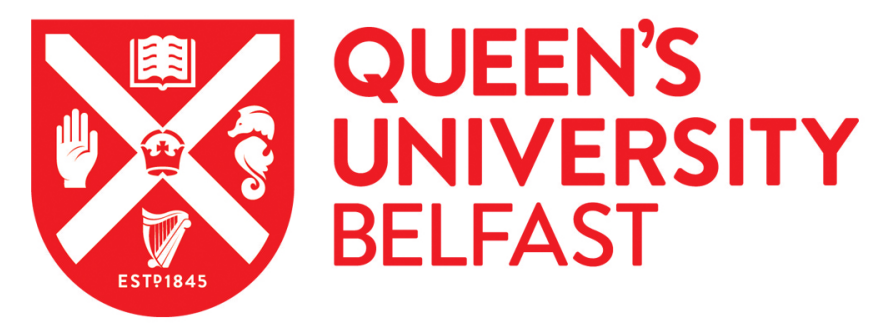

\title{
Non-Orthogonal Multiple Access Schemes with Partial Relay Selection
}

Lee, S., da Costa, D. B., Vien, Q-T., Duong, T. Q., \& de Sousa Jr, R. T. (2016). Non-Orthogonal Multiple Access Schemes with Partial Relay Selection. IET Communications. https://doi.org/10.1049/iet-com.2016.0836

\author{
Published in: \\ IET Communications
}

\section{Document Version:}

Peer reviewed version

Queen's University Belfast - Research Portal:

Link to publication record in Queen's University Belfast Research Portal

\section{Publisher rights}

Copyright 2016 Institution of Engineering and Technology

This paper is a postprint of a paper submitted to and accepted for publication in [journal] and is subject to Institution of Engineering and Technology Copyright. The copy of record is available at the IET Digital Library

\section{General rights}

Copyright for the publications made accessible via the Queen's University Belfast Research Portal is retained by the author(s) and / or other copyright owners and it is a condition of accessing these publications that users recognise and abide by the legal requirements associated with these rights.

\section{Take down policy}

The Research Portal is Queen's institutional repository that provides access to Queen's research output. Every effort has been made to ensure that content in the Research Portal does not infringe any person's rights, or applicable UK laws. If you discover content in the Research Portal that you believe breaches copyright or violates any law, please contact openaccess@qub.ac.uk. 


\title{
Non-Orthogonal Multiple Access Schemes with Partial Relay Selection
}

\author{
Sunyoung Lee, Daniel Benevides da Costa, Quoc-Tuan Vien, Trung Q. Duong, \\ and Rafael Timóteo de Sousa Jr.
}

\begin{abstract}
In this paper, non-orthogonal multiple access (NOMA) in amplify-and-forward relay systems with partial relay selection (PRS) is investigated. More specifically, new exact closed-form expressions for the outage probabilities at two users are derived, based on which an asymptotic analysis at high signalto-noise ratio (SNR) is carried out. Additionally, in order to investigate the performance gap between the NOMA and orthogonal multiple access (OMA) schemes, a closed-form approximate expression at high SNR for the sum rate is derived. Furthermore, relying on our results, the impact of the PRS on the sum rate and outage probability of the proposed NOMA scheme is examined. In particular, the derived asymptotic expressions show that the proposed scheme can improve over the traditional OMA not only the sum rate but also the user fairness. Finally, simulation results are presented to corroborate the analytical results.
\end{abstract}

\section{Index Terms}

Non-orthogonal multiple access (NOMA), spectrum efficiency, amplify-and-forward (AF) relay, partial relay selection, outage probability, sum rate.

\section{INTRODUCTION}

Non-orthogonal multiple access (NOMA) has recently received great attention from the wireless community as a promising technique to achieve enhanced spectrum efficiency, improved

S. Lee and T. Q. Duong are with Queen's University Belfast, U.K. (email: \{slee40, trung.q.duong\}@qub.ac.uk).

D. B. da Costa is with Federal University of Ceará, Brazil (email: danielbcosta@ieee.org).

Q.-T. Vien is with Middlesex University, U.K. (email: q.vien@mdx.ac.uk).

R. T. de Sousa Jr. is with University of Brasília, Brazil (email: desousa@unb.br).

Part of this paper was accepted for presentation at the IEEE International Symposium on Personal, Indoor and Mobile Radio Communications (PIMRC), Sep. 2016, Valencia, Spain. 
cell-edge user throughput and low transmit latency. With NOMA, multiple users can share both time and frequency resources by adjusting their power allocation ratio. Particularly, the users with better channel conditions first remove the messages intended for other users by applying successive interference cancellation (SIC) and then decode their own messages [1].

NOMA concept can be employed in a range of wireless systems and has been recently extended to many applications. In [2], a design of NOMA for the uplink transmission, which allows multiple users to share the same sub-carrier without any coding or spreading redundancy, was proposed. It was shown that the proposed uplink NOMA scheme can achieve bit error rate that is very close to that of orthogonal frequency-division multiple access (OFDMA) systems but with higher spectrum efficiency. The performance of the NOMA with randomly deployed users was analyzed in [3], where although the developed NOMA revealed to enhance the ergodic sum rates, its outage performance depended critically on the choices of the users targeted data rates and allocated powers. In [4], the application of the NOMA in large-scale underlay cognitive radio networks with randomly deployed users was studied employing a stochastic geometry approach. NOMA was also proposed in [5], [6] for multiple-input multiple-output (MIMO) systems. Furthermore, NOMA was also extended in [7] to a cooperative scenario, where users under good channel conditions can be used as relays for other users under poor channel conditions. Very recently, full duplex cooperative NOMA system was also proposed in $[8]$.

Among the promising wireless communications techniques, relay-based networks have arisen as an emerging scenario thanks to their benefits over single-hop networks, such as increasing the signal reliability, extending the radio coverage, and improving the system throughput, while using low power levels at the transmitter and employing a small number of antennas at the terminals [9]-[11]. The key idea is that the mobile users relay signals to each other in order to emulate an antenna array. Depending on the complexity of the relays, relay-based networks can be basically categorized into two main classes, namely decode-and-forward (DF) and amplify-and-forward (AF) [12], [13].

Owing to the fact that the deployment of multiple relays offers even more significant performance improvements in dual-hop cooperative networks, a special attention has been given to the analysis of relay selection techniques. In particular, along the last decade two popular relay selection strategies have been investigated, including opportunistic relay selection (ORS) [14] and partial relay selection (PRS) [15]. Unlike the ORS which is performed considering the channel 
state information (CSI) of both source-to-relay link (i.e., first-hop link) and relay-to-destination link (i.e., second-hop link), the PRS works with the knowledge of the CSI of only either the first-hop link or the second-hop link. Based on these pioneering papers, several other works have analyzed the system performance of dual-hop cooperative networks with the PRS. Specifically, a closed-form expression for the outage probability was derived in [16], asymptotic capacity bounds were developed in [17], and closed-form approximations for the ergodic capacity were presented in [18].

From the benefits of both relay networks and NOMA concept, it is promising to jointly develop NOMA under a relay network context. Particularly, a cooperative NOMA with simultaneous wireless information and power transfer (SWIPT) has been very recently studied in [19], where NOMA users located near the source act as energy harvesting relays to help far NOMA users. Different from the cooperative NOMA [7], a cooperative relay system employing the NOMA scheme was proposed in [20] and [21] to improve the spectral efficiency. Recently, the impact of relay selection for cooperative NOMA was studied in [22]. The design of NOMA with one relay was also studied in [23], [24]. However, to the best of our knowledge, previous works on the NOMA for cooperative relay systems considered solely single-relay networks so that the benefits of NOMA in multi-relay networks still remain to be investigated.

In this work, in order to fill partly the gap which exists in the literature about the use of NOMA in a relay network context, we investigate a new scheme assuming joint NOMA and PRS. Only AF relay is considered ${ }^{1}$. We also consider PRS scheme since it needs the knowledge of the CSI of only one-hop link. Thus, the use of AF relay in a PRS scheme is highly desirable in practice when complexity issues arise as a main concern. Based on the described system model, the outage performance and the sum rate of NOMA schemes in AF relay systems with PRS are investigated. The main contributions of this paper can be summarized as follows:

- NOMA with PRS is proposed to improve both sum rate and user fairness. Compared to the traditional OMA which mainly focuses on the maximization of sum rate and compared to the conventional NOMA which addresses a balanced trade-off between sum rate and user fairness, our proposed scheme can enhance both sum rate and user fairness.

- Outage probability and its asymptotic analysis are presented for the proposed schemes

\footnotetext{
${ }^{1}$ Although the DF relay gives better performance compared to the AF one, the former has higher complexity than the latter. Thus, due to complexity issues, in this paper we opt to consider only AF relays in the proposed system.
} 
to realize the impact of PRS on the performance of NOMA scheme. It is shown that performance gain can be achieved significantly by increasing the number of relays from one to two. In addition, the outage probability is shown to decrease significantly with a proper power allocation to users subject to a minimum desired signal-to-noise ratio (SNR).

- An approximation closed-form expression for the ergodic capacity of each user is derived for the proposed scheme. In this case, the impact of PRS on the sum rate performance of the NOMA scheme is examined. It is shown that the ergodic capacity of a user under inferior channel can be improved by properly selecting the power allocation ratio of users, while that of a user under superior channel can benefit from both the number of relays and the power allocation ratio.

- Several other insights are attained from our results. For instance, it is shown that the outage probabilities at users scale inversely with the product of the SNRs of the source-todestination and relay-to-destination links.

The rest of this paper is organized as follows. The system model for NOMA with PRS is presented in Section II. New closed-form expressions for the outage probability and their respective asymptotic expressions are derived in Section III. In Section IV, the sum rate of the proposed scheme is investigated. Numerical and simulation results are presented in Section V, which is followed by conclusions in Section VI.

\section{SYSTEM MODEL}

We consider a downlink cooperative dual-hop relay system as shown in Fig. 1 where a base station (BS) (i.e., S) intends to transmit the signal to two users (i.e., $D_{1}$ and $D_{2}$ ) with the help of one out of $K$ AF relays (i.e., $\left\{\mathrm{R}_{1}, \ldots, \mathrm{R}_{K}\right\}, K>1$ ). As aforementioned, the selected relay can be determined through some selection criteria, such as PRS or ORS. In this paper, we only consider the case of the PRS. For mathematical tractability, a homogeneous network topology is considered, where all wireless links exhibit frequency non-selective Rayleigh block fading and additive white Gaussian noise (AWGN).

In Fig. 1, the complex channel coefficient between $A$ and $B,\{A, B\} \in\left\{S, R_{k}, D_{i}\right\}, A \neq B$, $k=1, \ldots, K, i=1,2$, is denoted by $h_{\mathrm{AB}} \sim \mathcal{C N}\left(0, \Omega_{\mathrm{AB}}\right)$. The AWGN between $\mathrm{S}$ and $\mathrm{R}_{k}, \mathrm{R}_{k}$ and $\mathrm{D}_{i}$, and $\mathrm{S}$ and $\mathrm{D}_{i}$ are denoted by $n_{\mathrm{R}_{k}}, n_{\mathrm{R}_{k} \mathrm{D}_{i}}$, and $n_{\mathrm{D}_{i}}$, respectively. Without loss of generality, we assume that the channels of two users are ordered as $h_{\mathrm{SD}_{1}} \leq h_{\mathrm{SD}_{2}}$, and $n_{\mathrm{R}_{k}}, n_{\mathrm{R}_{k} \mathrm{D}_{i}}, n_{\mathrm{D}_{i}} \sim$ $\mathcal{C N}\left(0, N_{0}\right)$. We also consider that $\mathrm{D}_{1}$ and $\mathrm{D}_{2}$ are paired together to perform NOMA downlink 


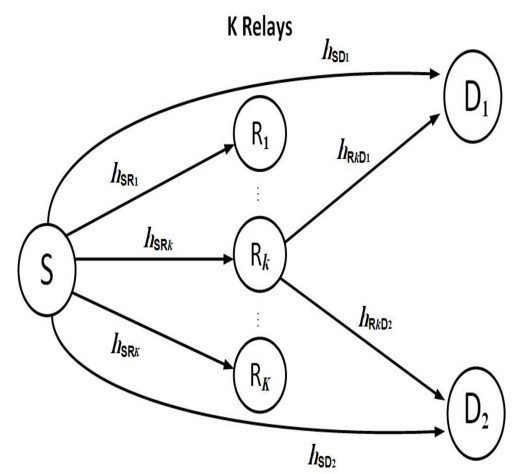

Fig. 1: The NOMA downlink cooperative dual-hop relay system.

cooperative system. Therefore, two consecutive phases are involved to complete the information transmission.

In the first phase, $\mathrm{S}$ broadcasts the information $x_{\mathrm{S}}$ to $\left\{\mathrm{R}_{k}\right\}, k=1, \ldots, K$ and both $\mathrm{D}_{1}$ and $\mathrm{D}_{2}$, which is given by

$$
x_{\mathrm{S}}=\sqrt{a_{1} P_{\mathrm{S}}} x_{1}+\sqrt{a_{2} P_{\mathrm{S}}} x_{2}
$$

where $a_{1}$ and $a_{2}$ are the power allocation coefficients, $x_{1}$ and $x_{2}$ are the messages for $\mathrm{D}_{1}$ and $\mathrm{D}_{2}$, respectively, and $P_{\mathrm{S}}$ is the transmit power at the BS. Following the principle of NOMA, we assume that $\left|a_{1}\right|>\left|a_{2}\right|$ with $a_{1}+a_{2}=1$. The received signals at $\mathrm{R}_{k}, \mathrm{D}_{1}$, and $\mathrm{D}_{2}$ are, respectively, given by

$$
\begin{aligned}
y_{\mathrm{R}_{k}} & =h_{\mathrm{SR}_{k}} x_{\mathrm{S}}+n_{\mathrm{R}_{k}} \\
& =h_{\mathrm{SR}_{k}}\left(\sqrt{a_{1} P_{\mathrm{S}}} x_{1}+\sqrt{a_{2} P_{\mathrm{S}}} x_{2}\right)+n_{\mathrm{R}_{k}}, \\
y_{\mathrm{D}_{1}} & =h_{\mathrm{SD}_{1}}\left(\sqrt{a_{1} P_{\mathrm{S}}} x_{1}+\sqrt{a_{2} P_{\mathrm{S}}} x_{2}\right)+n_{\mathrm{D}_{1}}, \\
y_{\mathrm{D}_{2}} & =h_{\mathrm{SD}_{2}}\left(\sqrt{a_{1} P_{\mathrm{S}}} x_{1}+\sqrt{a_{2} P_{\mathrm{S}}} x_{2}\right)+n_{\mathrm{D}_{2}} .
\end{aligned}
$$

In the second phase, $\mathrm{R}_{k}$ transmits the signal $x_{\mathrm{R}_{k}}=G_{k} y_{\mathrm{R}_{k}}$ to both $\mathrm{D}_{1}$ and $\mathrm{D}_{2}$, with $G_{k}$ denoting the amplifying gain at the $k$-th relay, i.e.,

$$
G_{k}^{2}=\frac{P_{\mathrm{R}_{k}}}{P_{\mathrm{S}}\left|h_{\mathrm{SR}_{k}}\right|^{2}+N_{0}},
$$

where $P_{\mathrm{R}_{k}}$ denotes the transmit power of the $k$-th relay. Without loss of generality, we assume that the transmit powers at all relays equal to the transmit power of the BS, i.e., $P_{\mathrm{R}_{1}}=P_{\mathrm{R}_{2}}=$ 
$\ldots=P_{\mathrm{R}_{K}}=P_{\mathrm{S}}=P$. Therefore, the received signals at $\mathrm{D}_{1}$ and $\mathrm{D}_{2}$ (forwarded by $\mathrm{R}_{k}$ ) are given by

$$
\begin{aligned}
y_{\mathrm{R}_{k} \mathrm{D}_{1}} & =h_{\mathrm{R}_{k} \mathrm{D}_{1}} x_{R_{k}}+n_{\mathrm{R}_{k} \mathrm{D}_{1}}=G_{k} h_{\mathrm{R}_{k} \mathrm{D}_{1}} h_{\mathrm{SR}_{k}} \sqrt{a_{1} P} x_{1} \\
& +G_{k} h_{\mathrm{R}_{k} \mathrm{D}_{1}} h_{\mathrm{SR}_{k}} \sqrt{a_{2} P} x_{2}+G_{k} h_{\mathrm{R}_{k} \mathrm{D}_{1}} n_{\mathrm{R}_{k}}+n_{\mathrm{R}_{k} \mathrm{D}_{1}}, \\
y_{\mathrm{R}_{k} \mathrm{D}_{2}} & =h_{\mathrm{R}_{k} \mathrm{D}_{2}} x_{R_{k}}+n_{\mathrm{R}_{k} \mathrm{D}_{2}}=G_{k} h_{\mathrm{R}_{k} \mathrm{D}_{2}} h_{\mathrm{SR}_{k}} \sqrt{a_{1} P} x_{1} \\
& +G_{k} h_{\mathrm{R}_{k} \mathrm{D}_{2}} h_{\mathrm{SR}_{k}} \sqrt{a_{2} P} x_{2}+G_{k} h_{\mathrm{R}_{k} \mathrm{D}_{2}} n_{\mathrm{R}_{k}}+n_{\mathrm{R}_{k} \mathrm{D}_{2}} .
\end{aligned}
$$

To calculate the instantaneous signal-to-interference-plus-noise ratio (SINR) of the two phases, let us define the average transmit SNR $\gamma_{0}=\frac{P}{N_{0}}$, and the random variables (RVs) $X_{i}=\gamma_{0}\left|h_{\mathrm{SD}_{i}}\right|^{2}$, $Y_{k}=\gamma_{0}\left|h_{\mathrm{SR}_{k}}\right|^{2}$ and $Z_{i k}=\gamma_{0}\left|h_{\mathrm{R}_{k} \mathrm{D}_{i}}\right|^{2}$ represent the instantaneous SNRs of the links $\mathrm{S} \rightarrow \mathrm{D}_{i}$, $\mathrm{S} \rightarrow \mathrm{R}_{k}$ and $\mathrm{R}_{k} \rightarrow \mathrm{D}_{i}$, respectively, where $k=1,2, \ldots, K$ and $i=1,2$.

In the first phase, treating $x_{2}$ as interference in $y_{D_{1}}$, the instantaneous SINR at $D_{1}$ can be given by

$$
\gamma_{\mathrm{SD}_{1}}=\frac{a_{1} \gamma_{0}\left|h_{\mathrm{SD}_{1}}\right|^{2}}{a_{2} \gamma_{0}\left|h_{\mathrm{SD}_{1}}\right|^{2}+1}=\frac{a_{1} X_{1}}{a_{2} X_{1}+1} .
$$

Similarly, the instantaneous SINR at $\mathrm{D}_{2}$ to detect $x_{1}$ can be written as

$$
\gamma_{\mathrm{SD}_{12}}=\frac{a_{1} X_{2}}{a_{2} X_{2}+1}
$$

Based on NOMA scheme, $D_{2}$ first decodes the message designated for $D_{1}$ and removes it using SIC, then it decodes its own message without interference. Therefore, the instantaneous SNR at $\mathrm{D}_{2}$ can be expressed as

$$
\gamma_{\mathrm{SD}_{2}}=a_{2} X_{2}
$$

In the second phase, the calculation of the instantaneous SINRs is similar to that in the first phase. Thus, the instantaneous SINR at $D_{1}$ related to link $R_{k} \rightarrow D_{1}$ can be written as

$$
\gamma_{\mathrm{R}_{k} \mathrm{D}_{1}}=\frac{a_{1} Y_{k} Z_{1 k}}{a_{2} Y_{k} Z_{1 k}+Y_{k}+Z_{1 k}+1} .
$$

Regarding the link $\mathrm{R}_{k} \rightarrow \mathrm{D}_{2}$, the instantaneous SINR at $\mathrm{D}_{2}$ to detect $x_{1}$ and the instantaneous SNR at $D_{2}$ to detect its own data $x_{2}$ are, respectively, given by

$$
\begin{gathered}
\gamma_{\mathrm{R}_{k} \mathrm{D}_{12}}=\frac{a_{1} Y_{k} Z_{2 k}}{a_{2} Y_{k} Z_{2 k}+Y_{k}+Z_{2 k}+1}, \\
\gamma_{\mathrm{R}_{k} \mathrm{D}_{2}}=\frac{a_{2} Y_{k} Z_{2 k}}{Y_{k}+Z_{2 k}+1} .
\end{gathered}
$$


Finally, from (8)-(13), the instantaneous SINRs at $D_{1}$ and $D_{2}$ based on selection combining, which has a lower complexity than maximal-ratio combining (MRC), can be written as

$$
\begin{aligned}
\gamma_{\mathrm{D}_{1}} & =\max \left(\gamma_{\mathrm{SD}_{1}}, \gamma_{\mathrm{R}_{k} \mathrm{D}_{1}}\right), \\
\gamma_{\mathrm{D}_{2}} & =\max \left(\gamma_{\mathrm{SD}_{2}}, \gamma_{\mathrm{R}_{k} \mathrm{D}_{2}}\right) .
\end{aligned}
$$

In the following section, we will calculate the outage probabilities of the two users employing a PRS strategy. Therefore, the selected relay index and its respective SNR are given by

$$
k^{*}=\arg \max _{k=1, . ., K} \gamma_{\mathrm{SR}_{k}}, \quad Y_{k^{*}}=\max _{k=1, . ., K} Y_{k}
$$

where $\gamma_{\mathrm{SR}_{k}}=Y_{k}$ denotes the instantaneous SNR at the $k$-th relay.

\section{Outage Probability Analysis}

The target SINRs of the two users are determined by their quality-of-service (QoS) requirements, of which each has its own preset target SINR, $\gamma_{t h_{i}}, i=1,2$. Keeping this in mind, in this section, we will derive the outage probabilities for the two paired users $D_{1}$ and $D_{2}$. For simplicity, we assume equal preset target SINRs of both $D_{1}$ and $D_{2}$, i.e., $\gamma_{t h_{1}}=\gamma_{t h_{2}}=\gamma_{t h}$.

\section{A. Outage Probability at $\mathrm{D}_{1}$}

According to [15], the cumulative distribution functions (CDFs) of the RVs $X_{i}, Y_{k^{*}}$ and $Z_{i k^{*}}$ are, respectively, given by

$$
\begin{aligned}
F_{X_{i}}\left(\gamma_{t h}\right) & =1-\exp \left(-\frac{\gamma_{t h}}{\widetilde{\Omega}_{\mathrm{SD}_{i}}}\right), \\
F_{Y_{k^{*}}}\left(\gamma_{t h}\right) & =\left(1-\exp \left(-\frac{\gamma_{t h}}{\widetilde{\Omega}_{\mathrm{SR}_{k^{*}}}}\right)\right)^{K} \\
& =1-\sum_{k=1}^{K}\left(\begin{array}{c}
K \\
k
\end{array}\right)(-1)^{k-1} \exp \left(-\frac{\gamma_{t h}}{\widetilde{\Omega}_{\mathrm{SR}_{k^{*}}}}\right), \\
F_{Z_{i k^{*}}}\left(\gamma_{t h}\right) & =1-\exp \left(-\frac{\gamma_{t h}}{\widetilde{\Omega}_{\mathrm{R}_{k^{*} \mathrm{D}_{i}}}}\right),
\end{aligned}
$$

where $\widetilde{\Omega}_{\mathrm{SD}_{i}}=\gamma_{0} \Omega_{\mathrm{SD}_{i}}, \widetilde{\Omega}_{\mathrm{SR}_{k^{*}}}=\gamma_{0} \Omega_{\mathrm{SR}_{k^{*}}}$, and $\widetilde{\Omega}_{\mathrm{R}_{k^{*}} \mathrm{D}_{i}}=\gamma_{0} \Omega_{\mathrm{R}_{k^{*}} \mathrm{D}_{i}}$ represent the average SNRs of the links $\mathrm{S} \rightarrow \mathrm{D}_{i}, \mathrm{~S} \rightarrow \mathrm{R}_{k^{*}}$ and $\mathrm{R}_{k^{*}} \rightarrow \mathrm{D}_{i}$, respectively. 
Based on the NOMA scheme, an outage event occurs if neither the direct transmission nor the relaying transmission succeeds. Therefore, the outage probability at $D_{1}$ can be expressed as

$$
\begin{aligned}
O P_{1} & =\operatorname{Pr}\left(\gamma_{\mathrm{D}_{1}}<\gamma_{t h}\right) \\
& =\operatorname{Pr}\left(\max \left[\gamma_{\mathrm{SD}_{1}}, \gamma_{\mathrm{R}_{k^{*}} \mathrm{D}_{1}}\right]<\gamma_{t h}\right) \\
& =F_{\gamma_{\mathrm{SD}_{1}}}\left(\gamma_{t h}\right) F_{\gamma_{\mathrm{R}^{*} \mathrm{D}_{1}}}\left(\gamma_{t h}\right),
\end{aligned}
$$

where we have

$$
\begin{aligned}
F_{\gamma_{\mathrm{SD}_{1}}}\left(\gamma_{t h}\right) & =\operatorname{Pr}\left(\frac{a_{1} X_{1}}{a_{2} X_{1}+1}<\gamma_{t h}\right) \\
& =\operatorname{Pr}\left(a_{1} X_{1}-a_{2} \gamma_{t h} X_{1}<\gamma_{t h}\right)
\end{aligned}
$$

and

$$
\begin{aligned}
F_{\gamma_{\mathrm{R}^{*} \mathrm{D}_{1}}}\left(\gamma_{t h}\right) & =\operatorname{Pr}\left(\frac{a_{1} Y_{k^{*}} Z_{1 k^{*}}}{a_{2} Y_{k^{*}} Z_{1 k^{*}}+Y_{k^{*}}+Z_{1 k^{*}}+1}<\gamma_{t h}\right) \\
& =\operatorname{Pr}\left(Y_{k^{*}}\left(Z_{1 k^{*}}\left(a_{1}-a_{2} \gamma_{t h}\right)-\gamma_{t h}\right)<Z_{1 k^{*}} \gamma_{t h}+\gamma_{t h}\right)
\end{aligned}
$$

As can be seen from (21), the outage always occurs if $a_{1}-a_{2} \gamma_{t h} \leq 0$, i.e., $a_{1} \leq a_{2} \gamma_{t h}$. Hence, we need to allocate more power to $D_{1}$, i.e., $a_{1}>a_{2} \gamma_{t h}$ is required to always hold. Based on (17), (21) can be rewritten as

$$
\begin{aligned}
F_{\gamma_{\mathrm{SD}_{1}}}\left(\gamma_{t h}\right) & =\operatorname{Pr}\left(X_{1}<\frac{\gamma_{t h}}{a_{1}-a_{2} \gamma_{t h}}=\tilde{\gamma}_{t h}\right) \\
& =F_{X_{1}}\left(\tilde{\gamma}_{t h}\right)=1-\exp \left(-\frac{\tilde{\gamma}_{t h}}{\widetilde{\Omega}_{\mathrm{SD}_{1}}}\right),
\end{aligned}
$$

where $\tilde{\gamma}_{t h} \triangleq \frac{\gamma_{t h}}{a_{1}-a_{2} \gamma_{t h}}$. From (22), one can also see that if $Z_{1 k^{*}}\left(a_{1}-a_{2} \gamma_{t h}\right)-\gamma_{t h} \leq 0$, the outage always occurs, whereas if $Z_{1 k^{*}}\left(a_{1}-a_{2} \gamma_{t h}\right)-\gamma_{t h}>0$, i.e., $Z_{1 k^{*}}>\tilde{\gamma}_{t h}$ then the outage may occur or not. As a result, (22) can be rewritten as

$$
\begin{aligned}
F_{\gamma_{\mathrm{R}_{k} \mathrm{D}_{1}}}\left(\gamma_{t h}\right) & =F_{Z_{1 k^{*}}}\left(\tilde{\gamma}_{t h}\right)+\int_{\tilde{\gamma}_{t h}}^{+\infty} F_{Y_{k^{*}}}\left(\frac{z \gamma_{t h}+\gamma_{t h}}{z\left(a_{1}-a_{2} \gamma_{t h}\right)-\gamma_{t h}}\right) f_{Z_{1 k^{*}}}(z) d z \\
& =F_{Z_{1 k^{*}}}\left(\tilde{\gamma}_{t h}\right)+\int_{\tilde{\gamma}_{t h}}^{+\infty} F_{Y_{k^{*}}}\left(\frac{z+1}{\frac{z}{\tilde{\gamma}_{t h}}-1}\right) f_{Z_{1 k^{*}}}(z) d z \\
& =F_{Z_{1 k^{*}}}\left(\tilde{\gamma}_{t h}\right)+\int_{\tilde{\gamma}_{t h}}^{+\infty} F_{Y_{k^{*}}}\left(\frac{\tilde{\gamma}_{t h}(z+1)}{z-\tilde{\gamma}_{t h}}\right) f_{Z_{1 k^{*}}}(z) d z
\end{aligned}
$$


By replacing (18) and (19) in $(24), F_{\gamma_{\mathrm{R}^{*}} \mathrm{D}_{1}}\left(\gamma_{t h}\right)$ can be derived as

$$
F_{\gamma_{\mathrm{R}^{*} \mathrm{D}_{1}}}\left(\gamma_{t h}\right)=1-\sum_{k=1}^{K}\left(\begin{array}{c}
K \\
k
\end{array}\right)(-1)^{k-1} \exp \left[-\tilde{\gamma}_{t h}\left(\frac{k}{\widetilde{\Omega}_{\mathrm{SR}_{k^{*}}}}+\frac{1}{\widetilde{\Omega}_{\mathrm{R}_{k^{*}} \mathrm{D}_{1}}}\right)\right] 2 \sqrt{\alpha} K_{1}(2 \sqrt{\alpha}),
$$

where $\alpha \triangleq \frac{k \tilde{\gamma}_{t h}\left(1+\tilde{\gamma}_{t h}\right)}{\widetilde{\Omega}_{\mathrm{SR}_{k^{*}}} \widetilde{\Omega}_{\mathrm{R}_{k^{*}} \mathrm{D}_{1}}}$ and $K_{1}(\cdot)$ denotes the first-order modified Bessel function of the second kind [25, Eq. (9.6.22)]. The proof of (25) is referred to Appendix A.

Finally, by substituting (25) and (23) into (20), a closed-form expression for the outage probability at $D_{1}$ can be derived as

$$
O P_{1}=\left[1-\exp \left(-\frac{\tilde{\gamma}_{t h}}{\widetilde{\Omega}_{\mathrm{SD}_{1}}}\right)\right]\left(1-\sum_{k=1}^{K}\left(\begin{array}{c}
K \\
k
\end{array}\right)(-1)^{k-1} \exp \left[-\tilde{\gamma}_{t h}\left(\frac{k}{\widetilde{\Omega}_{\mathrm{SR}_{k^{*}}}}+\frac{1}{\widetilde{\Omega}_{\mathrm{R}_{k^{*}} \mathrm{D}_{1}}}\right)\right] 2 \sqrt{\alpha} K_{1}(2 \sqrt{\alpha})\right) .
$$

\section{B. Outage Probability at $\mathrm{D}_{2}$}

$D_{2}$ will be in outage when both the first phase and the second phase are in outage. Therefore, the outage probability at $D_{2}$ can be formulated as

$$
O P_{2}=F_{\gamma_{\mathrm{SD}_{2}}}\left(\gamma_{t h}\right) F_{\gamma_{\mathrm{R}_{k} * \mathrm{D}_{2}}}\left(\gamma_{t h}\right)
$$

Note that $D_{2}$ needs to first decode the signal of $D_{1}$ and then its own data. Therefore, the term $F_{\gamma_{\mathrm{SD}_{2}}}\left(\gamma_{t h}\right)$ in (27) can be derived as

$$
\begin{aligned}
F_{\gamma_{\mathrm{SD}_{2}}}\left(\gamma_{t h}\right) & =\operatorname{Pr}\left(\frac{a_{1} X_{2}}{a_{2} X_{2}+1}<\gamma_{t h}, a_{2} X_{2}<\gamma_{t h}\right) \\
& =\operatorname{Pr}\left(X_{2}<\tilde{\gamma}_{t h}, X_{2}<\gamma_{t h}^{\prime}\right) \\
& =\operatorname{Pr}\left(X_{2}<\max \left[\tilde{\gamma}_{t h}, \gamma_{t h}^{\prime}\right]\right) \\
& =F_{X_{2}}(\theta) \\
& =1-\exp \left(-\frac{\theta}{\widetilde{\Omega}_{\mathrm{SD}_{2}}}\right),
\end{aligned}
$$

where $\gamma_{t h}^{\prime} \triangleq \frac{\gamma_{t h}}{a_{2}}$ and $\theta \triangleq \max \left(\tilde{\gamma}_{t h}, \gamma_{t h}^{\prime}\right)$. In (27), $F_{\gamma_{\mathrm{R}_{k^{*}} \mathrm{D}_{2}}}\left(\gamma_{t h}\right)$ can be calculated as

$$
F_{\gamma_{\mathrm{R}^{*} \mathrm{D}_{2}}}\left(\gamma_{t h}\right)=1-\sum_{k=1}^{K}\left(\begin{array}{c}
K \\
k
\end{array}\right)(-1)^{k-1} \exp \left[-\theta\left(\frac{k}{\widetilde{\Omega}_{\mathrm{SR}_{k^{*}}}}+\frac{1}{\widetilde{\Omega}_{\mathrm{R}_{k^{*} \mathrm{D}_{2}}}}\right)\right] 2 \sqrt{\beta} K_{1}(2 \sqrt{\beta})
$$

where $\beta \triangleq \frac{k \theta(1+\theta)}{\widetilde{\Omega}_{\mathrm{SR}_{k^{*}}} \widetilde{\Omega}_{\mathrm{R}_{k^{*}} \mathrm{D}_{2}}}$. The detailed proof of (29) is also provided in Appendix B. 
Finally, a closed-form expression for $O P_{2}$ is given by

$O P_{2}=\left[1-\exp \left(-\frac{\theta}{\widetilde{\Omega}_{\mathrm{SD}_{2}}}\right)\right]\left(1-\sum_{k=1}^{K}\left(\begin{array}{c}K \\ k\end{array}\right)(-1)^{k-1} \exp \left[-\theta\left(\frac{k}{\widetilde{\Omega}_{\mathrm{SR}_{k^{*}}}}+\frac{1}{\widetilde{\Omega}_{\mathrm{R}_{k^{*} \mathrm{D}_{2}}}}\right)\right] 2 \sqrt{\beta} K_{1}(2 \sqrt{\beta})\right)$.

\section{Asymptotic Outage Probability}

Based on the preceding results, an asymptotic outage analysis for both $D_{1}$ and $D_{2}$ will be now carried out in order to evaluate the behaviour of $O P_{1}$ and $O P_{2}$, respectively, in the high-SNR regime.

1) Asymptotic Outage Probability at $\mathrm{D}_{1}$ : Using the McLaurin expression, we have that $e^{x} \simeq$ $1+x$ and $K_{1}(x) \simeq x^{-1}$ for small $x$. Therefore, it follows that

$$
2 \sqrt{\alpha} K_{1}(2 \sqrt{\alpha}) \simeq 1
$$

Recalling that $\widetilde{\Omega}_{\mathrm{SD}_{i}}=\gamma_{0} \Omega_{\mathrm{SD}_{i}}$, the following simplifications can be performed by

$$
\begin{gathered}
1-\exp \left[-\frac{\gamma_{t h}}{\left(a_{1}-a_{2} \gamma_{t h}\right) \widetilde{\Omega}_{\mathrm{SD}_{1}}}\right] \simeq \frac{\tilde{\gamma}_{t h}}{\widetilde{\Omega}_{\mathrm{SD}_{1}}}=\frac{\tilde{\gamma}_{t h}}{\gamma_{0} \Omega_{\mathrm{SD}_{1}}}, \\
\exp \left[-\tilde{\gamma}_{t h}\left(\frac{k}{\widetilde{\Omega}_{\mathrm{SR}_{k^{*}}}}+\frac{1}{\widetilde{\Omega}_{\mathrm{R}_{k^{*} \mathrm{D}_{1}}}}\right)\right] \simeq 1-\tilde{\gamma}_{t h}\left(\frac{k}{\left.\widetilde{\Omega}_{\mathrm{SR}_{k^{*}}}+\frac{1}{\widetilde{\Omega}_{\mathrm{R}_{k^{*} \mathrm{D}_{1}}}}\right) .} .\right.
\end{gathered}
$$

Therefore, as a part of $O P_{1}$ (see (26)), (24) can be approximated by

$$
\begin{aligned}
& \tilde{A}_{1} \simeq 1-\sum_{k=1}^{K}\left(\begin{array}{c}
K \\
k
\end{array}\right)(-1)^{k-1}\left[1-\left(\frac{k \tilde{\gamma}_{t h}}{\widetilde{\Omega}_{\mathrm{SR}_{k^{*}}}}+\frac{\tilde{\gamma}_{t h}}{\widetilde{\Omega}_{\mathrm{R}_{k^{*}} \mathrm{D}_{1}}}\right)\right] \\
& =1-\sum_{k=1}^{K}\left(\begin{array}{c}
K \\
k
\end{array}\right)(-1)^{k-1}+\sum_{k=1}^{K}\left(\begin{array}{c}
K \\
k
\end{array}\right)(-1)^{k-1} \tilde{\gamma}_{t h}\left(\frac{k}{\widetilde{\Omega}_{\mathrm{SR}_{k^{*}}}}+\frac{1}{\widetilde{\Omega}_{\mathrm{R}_{k^{*}} \mathrm{D}_{1}}}\right) \text {. }
\end{aligned}
$$

Note that $\sum_{k=1}^{K}\left(\begin{array}{c}K \\ k\end{array}\right)(-1)^{k-1}=1$. Recalling that $\widetilde{\Omega}_{\mathrm{SR}_{k^{*}}}=\gamma_{0} \Omega_{\mathrm{SR}_{k^{*}}}, \widetilde{\Omega}_{\mathrm{R}_{k^{*}} \mathrm{D}_{i}}=\gamma_{0} \Omega_{\mathrm{R}_{k^{*}} \mathrm{D}_{i}}$,

can be reduced to

$$
\tilde{A}_{1} \simeq \frac{\tilde{\gamma}_{t h}}{\gamma_{0}}\left[\sum_{k=1}^{K}\left(\begin{array}{c}
K \\
k
\end{array}\right)(-1)^{k-1}\left(\frac{k}{\Omega_{\mathrm{SR}_{k^{*}}}}+\frac{1}{\Omega_{\mathrm{R}_{k^{*}} \mathrm{D}_{1}}}\right)\right] .
$$

Finally, from (32) and (35), an asymptotic outage expression for $O P_{1}$ in (26) can be derived as

$$
O P_{1} \simeq A_{1}\left(\frac{\tilde{\gamma}_{t h}}{\gamma_{0}}\right)^{2}
$$


where $A_{1}$ is a constant and does not depend on $\gamma_{0}$, being expressed as

$$
A_{1}=\frac{1}{\Omega_{\mathrm{SD}_{1}}}\left[\sum_{k=1}^{K}\left(\begin{array}{l}
K \\
k
\end{array}\right)(-1)^{k-1}\left(\frac{k}{\Omega_{\mathrm{SR}_{k^{*}}}}+\frac{1}{\Omega_{\mathrm{R}_{k^{*}} \mathrm{D}_{1}}}\right)\right] .
$$

Based on (36), one can conclude that the diversity order at $D_{1}$ equals to two.

2) Asymptotic Outage Probability at $\mathrm{D}_{2}$ : Similar to the derivation of $O P_{1}$, an asymptotic outage expression for $O P_{2}$, whose exact analysis was presented in (30), can be derived as

$$
O P_{2} \simeq A_{2}\left(\frac{\theta}{\gamma_{0}}\right)^{2}
$$

where $A_{2}$ is given by

$$
A_{2}=\frac{1}{\Omega_{\mathrm{SD}_{2}}}\left[\sum_{k=1}^{K}\left(\begin{array}{c}
K \\
k
\end{array}\right)(-1)^{k-1}\left(\frac{k}{\Omega_{\mathrm{SR}_{k^{*}}}}+\frac{1}{\Omega_{\mathrm{R}_{k^{*}} \mathrm{D}_{2}}}\right)\right] .
$$

From (36), one can also conclude that the diversity order at $D_{2}$ equals to two. Also, it is clearly seen from (36) and (36) that the diversity order of the considered system does not depend on the number of relays.

\section{Sum Rate ANALYSis}

In this section, we turn our attention to the sum rate analysis for NOMA with PRS. We aim to derive a closed-form expression of the sum rate based on high SNR approximation. The sum rate of the considered system is given by $\bar{C}_{\text {Sum }}=\bar{C}_{1}+\bar{C}_{2}$, where $\bar{C}_{1}$ and $\bar{C}_{2}$ are ergodic capacities at $D_{1}$ and $D_{2}$, respectively.

\section{A. The Ergodic Capacity at $\mathrm{D}_{1}$}

Using the selection combining rule, the capacity at $D_{1}$ depends on the maximum SINR of both links $S$ to $D_{1}$ and $R_{k}$ to $D_{1}$. The instantaneous capacity and ergodic capacity at $D_{1}$ are thus written as

$$
\begin{aligned}
C_{1}= & \frac{1}{2} \log _{2}\left(1+\max \left[\gamma_{\mathrm{SD}_{1}}, \gamma_{\mathrm{R}_{k^{*}} \mathrm{D}_{1}}\right]\right) . \\
\bar{C}_{1} & =\mathbb{E}\left[\frac{1}{2} \log _{2}\left(1+W_{1}\right)\right] \\
& =\frac{1}{2 \ln 2} \int_{0}^{\infty} \frac{1-F_{W_{1}}(x)}{1+x} d x,
\end{aligned}
$$


where $W_{1} \triangleq \max \left[\gamma_{\mathrm{SD}_{1}}, \gamma_{\mathrm{R}_{k} * \mathrm{D}_{1}}\right]$ and

$$
\begin{aligned}
F_{W_{1}}(x) & =\operatorname{Pr}\left(\max \left[\gamma_{\mathrm{SD}_{1}}, \gamma_{\mathrm{R}_{k^{*}} \mathrm{D}_{1}}\right]<x\right) \\
& =F_{\gamma_{\mathrm{SD}_{1}}}(x) F_{\gamma_{\mathrm{R}^{*} \mathrm{D}_{1}}}(x) \\
& =\left[1-\exp \left(-\frac{x}{\left(a_{1}-a_{2} x\right) \widetilde{\Omega}_{\mathrm{SD}_{1}}}\right)\right]\left[1-\sum_{k=1}^{K}\left(\begin{array}{c}
K \\
k
\end{array}\right)(-1)^{k-1}\right. \\
& \left.\times \exp \left(-\frac{x}{a_{1}-a_{2} x}\left(\frac{k}{\widetilde{\Omega}_{\mathrm{SR}_{k^{*}}}}+\frac{1}{\widetilde{\Omega}_{\mathrm{R}_{k^{*} \mathrm{D}_{1}}}}\right)\right) 2 \sqrt{\alpha} K_{1}(2 \sqrt{\alpha})\right] .
\end{aligned}
$$

By replacing (42) in (41), the ergodic capacity at $D_{1}$ can be derived as

$$
\bar{C}_{1}(x)=\frac{1}{2 \ln 2}\left[\int_{0}^{\infty} \frac{\exp \left(-\frac{x}{\left(a_{1}-a_{2} x\right) \widetilde{\Omega}_{\mathrm{SD}_{1}}}\right)}{1+x} d x+\sum_{k=1}^{K}\left(\begin{array}{c}
K \\
k
\end{array}\right)(-1)^{k-1} \varphi\right] \text {, }
$$

where

$$
\varphi=\int_{0}^{\infty} \frac{\left(1-\exp \left(-\frac{x}{\left(a_{1}-a_{2} x\right) \tilde{\Omega}_{\mathrm{SD}_{1}}}\right)\right) \exp \left(-\frac{x}{a_{1}-a_{2} x}\left(\frac{k}{\Omega_{\mathrm{SR}_{k^{*}}}}+\frac{1}{\Omega_{\mathrm{R}_{k^{*}} \mathrm{D}_{1}}}\right)\right)}{1+x} 2 \sqrt{\alpha} K_{1}(2 \sqrt{\alpha}) d x .
$$

Using the exponential integral function, (43) can be re-written by

$$
\begin{aligned}
\bar{C}_{1}(x)=\frac{1}{2 \ln 2} & {\left[\exp \left(\frac{1}{a_{2} \widetilde{\Omega}_{\mathrm{SD}_{1}}}\right) \mathrm{E}_{\mathrm{i}}\left(-\frac{1}{a_{2} \widetilde{\Omega}_{\mathrm{SD}_{1}}}\right)-\exp \left(\frac{1}{\widetilde{\Omega}_{\mathrm{SD}_{1}}}\right) \mathrm{E}_{\mathrm{i}}\left(-\frac{1}{\widetilde{\Omega}_{\mathrm{SD}_{1}}}\right)\right.} \\
& \left.+\sum_{k=1}^{K}\left(\begin{array}{c}
K \\
k
\end{array}\right)(-1)^{k-1} \varphi\right],
\end{aligned}
$$

where $\mathrm{E}_{\mathrm{i}}(x)$ denotes the exponential integral function [26, Eq.(3.352.4)]. Note that (43) is the exact expression for the ergodic capacity at $D_{1}$ and it still contains an indefinite integral which is difficult to obtain in closed-form. To deal with this, we will focus on the high SNR approximation. Specifically, when $\gamma_{0} \rightarrow \infty$, the ergodic capacity can be expressed as follows

$$
\begin{aligned}
\bar{C}_{1} & =\mathbb{E}\left[\frac{1}{2} \log _{2}\left(1+\max \left[\frac{a_{1} X_{1}}{a_{2} X_{1}+1}, \frac{a_{1} Y_{k} Z_{1 k}}{a_{2} Y_{k} Z_{1 k}+Y_{k}+Z_{1 k}+1}\right]\right)\right] \\
& \approx \frac{1}{2} \log _{2}\left(1+\frac{a_{1}}{a_{2}}\right) .
\end{aligned}
$$




\section{B. The Ergodic Capacity at $\mathrm{D}_{2}$}

Based on NOMA scheme, the capacity at $D_{2}$ depends on the maximum SNR between $S$ to $\mathrm{D}_{2}$ and $\mathrm{R}_{k}$ to $\mathrm{D}_{2}$, if $\left|h_{\mathrm{R}_{k} \mathrm{D}_{1}}\right|^{2} \leq\left|h_{\mathrm{R}_{k} \mathrm{D}_{2}}\right|^{2}$. Otherwise, SNR from only direct link is considered due to SIC. Therefore, $C_{2}$ can be written as

$$
C_{2}= \begin{cases}\frac{1}{2} \log _{2}\left(1+\max \left[\gamma_{\mathrm{SD}_{2}}, \gamma_{\mathrm{R}_{k^{*}} \mathrm{D}_{2}}\right]\right), & \text { for }\left|h_{\mathrm{R}_{k} \mathrm{D}_{1}}\right|^{2} \leq\left|h_{\mathrm{R}_{k} \mathrm{D}_{2}}\right|^{2} \\ \frac{1}{2} \log _{2}\left(1+\gamma_{\mathrm{SD}_{2}}\right), & \text { for }\left|h_{\mathrm{R}_{k} \mathrm{D}_{1}}\right|^{2}>\left|h_{\mathrm{R}_{k} \mathrm{D}_{2}}\right|^{2} .\end{cases}
$$

Taking expectation of (47), the ergodic capacity at $D_{2}$ can be calculated as

$$
\begin{aligned}
\bar{C}_{2} & =\underbrace{\operatorname{Pr}\left(\left|h_{\mathrm{R}_{k} \mathrm{D}_{1}}\right|^{2} \leq\left|h_{\mathrm{R}_{k} \mathrm{D}_{2}}\right|^{2}\right)}_{I_{1}} \underbrace{\mathbb{E}\left[\frac{1}{2} \log _{2}\left(1+\max \left[\gamma_{\mathrm{SD}_{2}}, \gamma_{\mathrm{R}_{k^{*}} \mathrm{D}_{2}}\right]\right)\right]}_{J_{1}} \\
& +\underbrace{\operatorname{Pr}\left(\left|h_{\mathrm{R}_{k} \mathrm{D}_{1}}\right|^{2}>\left|h_{\mathrm{R}_{k} \mathrm{D}_{2}}\right|^{2}\right)}_{I_{2}} \underbrace{\mathbb{E}\left[\frac{1}{2} \log _{2}\left(1+\gamma_{\mathrm{SD}_{2}}\right)\right]}_{J_{2}} .
\end{aligned}
$$

Since $h_{\mathrm{R}_{k} \mathrm{D}_{1}}$ and $h_{\mathrm{R}_{k} \mathrm{D}_{2}}$ are independent and identically distributed (i.d.d.) RVs, it follows that $I_{1}=I_{2}=\frac{1}{2}$. The remaining parts $J_{1}$ and $J_{2}$ of the ergodic capacity at $\mathrm{D}_{2}$ are derived in the sequel. Particularly, we have

$$
\begin{aligned}
J_{1} & =\mathbb{E}\left[\frac{1}{2} \log _{2}\left(1+\max \left[\gamma_{\mathrm{SD}_{2}}, \gamma_{\mathrm{R}_{k^{*}} \mathrm{D}_{2}}\right]\right)\right] \\
& =\mathbb{E}\left[\frac{1}{2} \log _{2}\left(1+a_{2} W_{2}\right)\right] \\
& =\frac{a_{2}}{2 \ln 2} \int_{0}^{\infty} \frac{1-F_{W_{2}}(x)}{1+a_{2} x} d x,
\end{aligned}
$$

where $W_{2} \triangleq \max \left[X_{2}, \frac{a_{2} Y_{k^{*}} Z_{2 k^{*}}}{Y_{k^{*}}+Z_{2 k^{*}}+1}\right]$. An upper bound for $W_{2}$ can be found owing to the fact that $W_{2}<\max \left[X_{2}, \min \left[Y_{k^{*}}, Z_{2 k^{*}}\right]\right]$. Based on this, it follows that

$$
\begin{aligned}
F_{W_{2}}(x) & =\operatorname{Pr}\left(\max \left[X_{2}, \frac{Y_{k^{*}} Z_{2 k^{*}}}{Y_{k^{*}}+Z_{2 k^{*}}+1}\right]<x\right) \\
& \leq F_{X_{2}}(x) F_{\min \left[Y_{k^{*}}, Z_{2 k^{*}}\right]}(x) \\
& =F_{X_{2}}(x)\left[1-\left(1-F_{Y_{k^{*}}}(x)\right)\left(1-F_{Z_{2 k^{*}}}(x)\right)\right] \\
& =\left[1-\exp \left(-\frac{x}{\widetilde{\Omega}_{\mathrm{SD}_{2}}}\right)\right]\left[1-\sum_{k=1}^{K}\left(\begin{array}{c}
K \\
k
\end{array}\right)(-1)^{k-1} \exp \left(-x\left(\frac{k}{\widetilde{\Omega}_{\mathrm{SR}_{k^{*}}}}+\frac{1}{\widetilde{\Omega}_{\mathrm{R}_{k^{*} \mathrm{D}_{2}}}}\right)\right)\right] .
\end{aligned}
$$


Finally, by replacing (50) in (49), an upper bound for $J_{1}$ can be obtained in closed-form as

$$
\begin{aligned}
J_{1} \leq \frac{1}{2 \ln 2} \int_{0}^{\infty} \frac{1-\left[1-\exp \left(-\frac{x}{\Omega_{\mathrm{SD}_{2}}}\right)\right]\left[1-\sum_{k=1}^{K}\left(\begin{array}{c}
K \\
k
\end{array}\right)(-1)^{k-1} \exp \left(-x\left(\frac{k}{\Omega_{\mathrm{SR}_{k^{*}}}}+\frac{1}{\widetilde{\Omega}_{\mathrm{R}_{k^{*}} \mathrm{D}_{2}}}\right)\right)\right]}{\frac{1}{a_{2}}+x} d x \\
=\frac{1}{2 \ln 2}\left[-\exp \left(\frac{1}{a_{2} \widetilde{\Omega}_{\mathrm{SD}_{2}}}\right) \mathrm{E}_{\mathrm{i}}\left(-\frac{1}{a_{2} \widetilde{\Omega}_{\mathrm{SD}_{2}}}\right)+\sum_{k=1}^{K}\left(\begin{array}{c}
K \\
k
\end{array}\right)(-1)^{k-1}\left(\exp \left(\frac{\xi_{1}}{a_{2}}\right) \mathrm{E}_{\mathrm{i}}\left(-\frac{\xi_{1}}{a_{2}}\right)\right.\right. \\
\left.\left.\quad-\exp \left(\frac{\xi_{2}}{a_{2}}\right) \mathrm{E}_{\mathrm{i}}\left(-\frac{\xi_{2}}{a_{2}}\right)\right)\right]
\end{aligned}
$$

where $\xi_{1}=\left(\frac{1}{\widetilde{\Omega}_{\mathrm{SD}_{2}}}+\frac{k}{\widetilde{\Omega}_{\mathrm{SR}_{k^{*}}}}+\frac{1}{\widetilde{\Omega}_{\mathrm{R}_{k^{*}} \mathrm{D}_{2}}}\right)$ and $\xi_{2}=\left(\frac{k}{\widetilde{\Omega}_{\mathrm{SR}_{k^{*}}}}+\frac{1}{\widetilde{\Omega}_{\mathrm{R}_{k^{*}} \mathrm{D}_{2}}}\right)$. Similar to (49), a closedform upper bound for $J_{2}$ can be attained as

$$
\begin{aligned}
J_{2} & =\mathbb{E}\left[\frac{1}{2} \log _{2}\left(1+a_{2} X_{2}\right)\right] \\
& =\frac{a_{2}}{2 \ln 2} \int_{0}^{\infty} \frac{1-F_{X_{2}}(x)}{1+a_{2} x} d x \\
& \leq \frac{1}{2 \ln 2}\left(-\exp \left(\frac{1}{a_{2} \widetilde{\Omega}_{\mathrm{SD}_{2}}}\right)\right) \mathrm{E}_{\mathrm{i}}\left(-\frac{1}{a_{2} \widetilde{\Omega}_{\mathrm{SD}_{2}}}\right) .
\end{aligned}
$$

Now, by substituting (51) and (52) into (48), a closed-form expression for the ergodic capacity at $D_{2}$ can be derived as

$$
\begin{aligned}
\bar{C}_{2} & \leq \frac{1}{4 \ln 2}\left[-2 \exp \left(\frac{1}{a_{2} \widetilde{\Omega}_{\mathrm{SD}_{2}}}\right) \mathrm{E}_{\mathrm{i}}\left(-\frac{1}{a_{2} \widetilde{\Omega}_{\mathrm{SD}_{2}}}\right)+\sum_{k=1}^{K}\left(\begin{array}{c}
K \\
k
\end{array}\right)(-1)^{k-1}\right. \\
& \left.\times\left[\exp \left(\frac{\xi_{1}}{a_{2}}\right) \mathrm{E}_{\mathrm{i}}\left(-\frac{\xi_{1}}{a_{2}}\right)-\exp \left(\frac{\xi_{2}}{a_{2}}\right) \mathrm{E}_{\mathrm{i}}\left(-\frac{\xi_{2}}{a_{2}}\right)\right]\right] .
\end{aligned}
$$

\section{Sum Rate}

The sum rate can be obtained by summing (46) and (53). Particularly, considering the approximations $\mathrm{E}_{\mathrm{i}}(-x) \approx \mathrm{E}_{\mathrm{c}}+\ln (x)$, [26, Eq. (8.212.1)], where $\mathrm{E}_{\mathrm{c}}$ denotes Euler's constant defined by $\lim _{s \rightarrow \infty}\left(\sum_{m=1}^{s} \frac{1}{m}-\ln s\right)$, and $e^{x} \approx 1+x$ for small $x$, the sum rate at high SNR can be approximated by

$$
\begin{aligned}
\bar{C}_{\text {Sum }} & \approx \frac{1}{2} \log _{2}\left(1+\frac{a_{1}}{a_{2}}\right)+\frac{1}{4 \ln 2}\left[-2\left(1+\left(\frac{1}{a_{2} \widetilde{\Omega}_{\mathrm{SD}_{2}}}\right)\right)\left(\mathrm{E}_{\mathrm{c}}+\ln \left(\frac{1}{a_{2} \widetilde{\Omega}_{\mathrm{SD}_{2}}}\right)\right)\right. \\
& +\sum_{k=1}^{K}\left(\begin{array}{c}
K \\
k
\end{array}\right)(-1)^{k-1}\left[\left(1+\frac{\xi_{1}}{a_{2}}\right)\left(\mathrm{E}_{\mathrm{c}}+\ln \left(-\frac{\xi_{1}}{a_{2}}\right)\right)-\left(1+\frac{\xi_{2}}{a_{2}}\right)\left(\mathrm{E}_{\mathrm{c}}+\ln \left(\frac{\xi_{2}}{a_{2}}\right)\right)\right],
\end{aligned}
$$


where the first and the second terms correspond to $\bar{C}_{1}$ and $\bar{C}_{2}$, respectively. It can be found from (54) that, in the high-SNR regime, $\bar{C}_{2}$ increases log-linearly with increasing $\gamma_{0}$ from $\widetilde{\Omega}_{\mathrm{SD}_{2}}$, $\widetilde{\Omega}_{\mathrm{SR}_{k^{*}}}$ and $\widetilde{\Omega}_{\mathrm{R}_{k^{*}} \mathrm{D}_{2}}$, while $\bar{C}_{1}$ relies only on the power allocation ratio, i.e., $\frac{a_{1}}{a_{2}}$, converging to $\frac{1}{2} \log _{2}\left(1+\frac{a_{1}}{a_{2}}\right)$.

\section{Numerical Results AND Discussions}

In this section, representative numerical results are provided to illustrate the impact of relay selection on the performance of the considered NOMA schemes. Also, simulation results are presented and excellent agreement between the simulated and analytical curves is observed, which corroborates the proposed analysis. In the considered system, the power allocation parameters are set as $a_{1}=0.8$ and $a_{2}=0.2$. Also, for simplicity and without any loss of generality, we assume that the target SINRs at Users 1 and 2 are the same, $\gamma_{t h}=1 \mathrm{~dB}$.

Fig. 2 plots the outage performance of User 1 versus the average SNR in order to show the impact of relay. In this figure, focusing on the PRS scheme, we consider only relay link, i.e., the impact of direct link is neglected, for various values of the number of relays. e.g., $K=1,2,10$. Other parameters are set as $\Omega_{\mathrm{SR}}=1$ and $\Omega_{\mathrm{RD}}=10$. It is interesting to see from Fig. 2 that a significant performance gain can be achieved by increasing the number of relays from one to two relays. For example, to achieve an outage probability of $10^{-2}$, two relays provide a power gain of $8 \mathrm{~dB}$ over the one relay case. However, note that the achievable power gain for the same outage probabilities is reduced to $4 \mathrm{~dB}$ by comparing the cases of $K=2$ and $K=10$. Particularly, for high SNRs, there is almost no gain in outage performance when we increase the number of relays from two to ten. This important result implies that for the NOMA scheme with PRS, employing more than two relays is unnecessary.

In order to show the impact of the relay, Fig. 3 plots the outage performance of User 2 versus the average SNR assuming relay link only. In this figure, a similar behaviour with the outage probability of User 1 shown in Fig. 2 is observed, but the outage probabilities at User 1 and User 2 are plotted separately to avoid dense figure. For example, it is clearly shown in Fig. 3 that the outage probability of User 2 can be decreased by increasing the number of relays.

To compare the outage performance of the two users, the outage probabilities at User 1 and User 2 are depicted in Fig. 4 , assuming $K=3, \Omega_{\mathrm{SD}_{1}}=\Omega_{\mathrm{SD}_{2}}=\Omega_{\mathrm{SR}}=\Omega_{\mathrm{RD}_{1}}=\Omega_{\mathrm{RD}_{2}}=1$. In this figure, we consider both relay and direct links at each user. Fig. 4 shows that the outage probability of User 1 outperforms that of User 2, which verifies the analytical results in (26) and 
(30). Moreover, taking a closer look at the outage expressions, it can be seen that $O P_{1}$ always achieves the outage probability less than $O P_{2}$ because $\theta$ is bigger than $\tilde{\gamma}_{t h}$. Such behaviour is clearly shown in Fig. 4. In addition, the simulations results corroborate the tight accuracy of the derived analytical expressions.

In Fig. 5, the impact of relay selection in NOMA is depicted through the ergodic capacities of two users, when setting $K=3$ and $\Omega_{\mathrm{SD}_{1}}=\Omega_{\mathrm{SD}_{2}}=\Omega_{\mathrm{SR}}=\Omega_{\mathrm{RD}_{1}}=\Omega_{\mathrm{RD}_{2}}=1$. For comparison, simulation results are provided to validate the accuracy of the attained analytical results. Moreover, the ergodic capacities of the proposed system is compared to OMA-based system. In particular, it can be seen in this figure that the ergodic capacity of User 1 is higher than that of OMA-based system in the low and moderate SNR regions while User 2 achieved higher ergodic capacity than OMA-based one in the wide range of SNR. Interestingly, note that the achievable capacity for User 1 converges to a constant in the medium and high SNR regions because interferences in the instantaneous SINRs at User 1 increase as average SNR increase while that for User 2 increase with increasing SNR, which are shown in (46) and (53). Furthermore, we consider another case when the power allocation factors are set as $a_{1}=0.9$ and $a_{2}=0.1$, as shown in this figure. This result implies that for the user fairness, ergodic capacity of an user under inferior channel can be improved by properly selecting the power allocation ratio of users and shows the superiority of NOMA compared to OMA.

Fig. 6 shows the sum rate for the proposed system and its high SNR approximation versus average SNR with the same system setting in Fig. 5. As can be observed from Fig. 6, the sum rate of our system outperforms than that of OMA-based one in a wide range of SNRs, which demonstrates the motivation of NOMA. The figure also depicts that the analytical expression for the system sum rate is matched closely with the simulation result. Also, the approximated expression in (54) matches very well with the exact one in the medium and high SNR regions. According to the results presented in Figs. 5 and 6 , it is proven that the proposed system for NOMA with PRS can enhance both sum rate and user fairness by increasing the number of relays and adjusting the power allocation ratio between an user under inferior channel and an user under superior channel.

\section{Conclusions}

In this paper, the effect of partial relay selection on the performance of NOMA schemes was investigated. For the performance analysis, exact closed-form expressions and asymptotic 
expressions for the outage probabilities of the two users have been derived, based on which insightful conclusions have been attained. Also, new exact and approximate expressions for the sum rate of the proposed NOMA scheme with PRS have been derived. Through the asymptotic and numerical results, we have clearly presented that the ergodic capacity at inferior user relies only on the power allocation ratio in the high SNR regime while that at superior user increases with increasing SNR. Also, interestingly, the numerical results showed that the NOMA scheme with PRS improves the performance gain by increasing the number of relays, but there is almost no gain in outage performance when the number of relays increases from two to ten at high SNR. This implies that for NOMA schemes with PRS, it is unnecessary to use more than two relays. The derived analytical expressions have been validated through Monte Carlo simulations and will be useful to evaluate the performance of various concepts of future NOMA with PRS. As a future work, the case of multiple users arises as an interesting issue to be investigated.

\section{APPENDIX A}

In this appendix, the proof of (25) is concerned. First, replacing (18) in (24), $F_{\gamma_{\mathrm{R}^{*}} \mathrm{D}_{1}}\left(\gamma_{t h}\right)$ can be derived as

$$
F_{\gamma_{\mathrm{R}_{k^{*}} \mathrm{D}_{1}}}\left(\gamma_{t h}\right)=F_{Z_{1 k^{*}}}\left(\tilde{\gamma}_{t h}\right)+\int_{\tilde{\gamma}_{t h}}^{+\infty}\left[1-\sum_{k=1}^{K}\left(\begin{array}{c}
K \\
k
\end{array}\right)(-1)^{k-1} \exp \left(-\frac{k}{\widetilde{\Omega}_{\mathrm{SR}_{k^{*}}}}\left(\frac{\tilde{\gamma}_{t h}(z+1)}{z-\tilde{\gamma}_{t h}}\right)\right)\right] f_{Z_{1 k^{*}}}(z) d z,
$$

Then, replacing (19) in (55) and after simplification, $F_{\gamma_{\mathrm{R}_{k} * \mathrm{D}_{1}}}\left(\gamma_{t h}\right)$ can be re-written as

$$
\begin{aligned}
F_{\gamma_{\mathrm{R}^{*} \mathrm{D}_{1}}}\left(\gamma_{t h}\right) & =1-\sum_{k=1}^{K}\left(\begin{array}{c}
K \\
k
\end{array}\right)(-1)^{k-1} \frac{1}{\widetilde{\Omega}_{\mathrm{R}_{k^{*}} \mathrm{D}_{1}}} \int_{\tilde{\gamma}_{t h}}^{+\infty} \exp \left(-\frac{k}{\widetilde{\Omega}_{\mathrm{SR}_{k^{*}}}}\left(\frac{\tilde{\gamma}_{t h}(z+1)}{z-\tilde{\gamma}_{t h}}\right)\right) \exp \left(-\frac{z}{\widetilde{\Omega}_{\mathrm{R}_{k^{*}} \mathrm{D}_{1}}}\right) d z \\
& \stackrel{(a)}{=} 1-\sum_{k=1}^{K}\left(\begin{array}{c}
K \\
k
\end{array}\right)(-1)^{k-1} \exp \left[-\tilde{\gamma}_{t h}\left(\frac{k}{\widetilde{\Omega}_{\mathrm{SR}_{k^{*}}}}+\frac{1}{\widetilde{\Omega}_{\mathrm{R}_{k^{*}} \mathrm{D}_{1}}}\right)\right] 2 \sqrt{\alpha} K_{1}(2 \sqrt{\alpha}),
\end{aligned}
$$

where (a) is achieved by employing the first-order modified Bessel function of the second kind, $K_{1}(\cdot)$ [25, Eq. (9.6.22)].

\section{APPENDIX B}

In this appendix, we provide the proof of (29). To this end, notice that the second term of (27) will be outage if either the SINR at D2 or the SNR at D2 is less than or equal to $\gamma_{t} h$. 
Based on this, the second term can be written as

$$
\begin{aligned}
F_{\gamma_{\mathrm{R}^{*} \mathrm{D}_{2}}}\left(\gamma_{t h}\right) & =\operatorname{Pr}\left(\frac{a_{1} Y_{k^{*}} Z_{2 k^{*}}}{a_{2} Y_{k^{*}} Z_{2 k^{*}}+Y_{k^{*}}+Z_{2 k^{*}}+1}<\gamma_{t h}, \frac{a_{2} Y_{k^{*}} Z_{2 k^{*}}}{Y_{k^{*}}+Z_{2 k^{*}}+1}<\gamma_{t h}\right) \\
& =\operatorname{Pr}\left(Y_{k^{*}}<\frac{Z_{2 k^{*}}+1}{\frac{Z_{2 k^{*}}}{\tilde{\gamma}_{t h}}-1}, Y_{k^{*}}<\frac{Z_{2 k^{*}}+1}{\frac{Z_{2 k^{*}}}{\gamma_{t h}^{\prime}}-1}\right) .
\end{aligned}
$$

Recalling that $\theta \triangleq \max \left(\tilde{\gamma}_{t h}, \gamma_{t h}^{\prime}\right)$, (57) can be simplified as

$$
F_{\gamma_{R_{k^{*}} \mathrm{D}_{2}}}\left(\gamma_{t h}\right)=\operatorname{Pr}\left(Y_{k^{*}}<\frac{Z_{2 k^{*}}+1}{\frac{Z_{2 k^{*}}}{\theta}-1}\right)=F_{Z_{2 k^{*}}}(\theta)+\int_{\theta}^{+\infty} F_{Y_{k^{*}}}\left(\frac{\theta(z+1)}{z-\theta}\right) f_{Z_{2 k^{*}}}(z) d z .
$$

Replacing (18) in (58), $F_{\gamma_{\mathrm{R}^{*} \text { D }}}\left(\gamma_{t h}\right)$ can be derived as

$$
\begin{aligned}
F_{\gamma_{\mathrm{R}^{*} \mathrm{D}_{2}}}\left(\gamma_{t h}\right) & =F_{Z_{2 k^{*}}}(\theta)+\int_{\theta}^{+\infty}\left[1-\sum_{k=1}^{K}\left(\begin{array}{c}
K \\
k
\end{array}\right)(-1)^{k-1} \exp \left(-\frac{k}{\widetilde{\Omega}_{\mathrm{SR}_{k^{*}}}}\left(\frac{\theta(z+1)}{z-\theta}\right)\right)\right] f_{Z_{2 k^{*}}}(z) d z \\
& \stackrel{(a)}{=} 1-\sum_{k=1}^{K}\left(\begin{array}{c}
K \\
k
\end{array}\right)(-1)^{k-1} \frac{1}{\widetilde{\Omega}_{\mathrm{R}_{k^{*}} \mathrm{D}_{2}}} \int_{\theta}^{+\infty} \exp \left(-\frac{k}{\widetilde{\Omega}_{\mathrm{SR}_{k^{*}}}}\left(\frac{\theta(z+1)}{z-\theta}\right)\right) \exp \left(-\frac{z}{\widetilde{\Omega}_{\mathrm{R}_{k^{*}} \mathrm{D}_{2}}}\right) d z,
\end{aligned}
$$

where (a) can be obtained by using the first-order modified Bessel function of the second kind.

\section{REFERENCES}

[1] Y. Saito, Y. Kishiyama, A. Benjebbour, T. Nakamura, A. Li, and K. Higuchi, "Non-orthogonal multiple access (NOMA) for cellular future radio access," in Proc. of IEEE 77th Vehicular Technol. Conf. (VTC Spring), Dresden, Germany, Jun. 2013, pp. 1-5.

[2] M. Al-Imari, P. Xiao, M. A. Imran, and R. Tafazolli, "Uplink non-orthogonal multiple access for 5G wireless networks," in Proc. of Int. Symp. on Wireless Commun. Syst. (ISWCS), Barcelona, Spain, Aug. 2014, pp. 781-785.

[3] Z. Ding, Z. Yang, P. Fan, and H. V. Poor, "On the performance of non-orthogonal multiple access in 5G systems with randomly deployed users," IEEE Signal Process. Lett., vol. 21, no. 12, pp. 1501-1505, Dec. 2014.

[4] Y. Liu, Z. Ding, M. Elkashlan, and J. Yuan, "Non-orthogonal multiple access in large-scale underlay cognitive radio networks," IEEE Trans. Veh. Technol., vol. PP, no. 99, pp. 1-1, Feb. 2016.

[5] Z. Ding, F. Adachi, and H. V. Poor, "Performance of MIMO-NOMA downlink transmissions," in Proc. IEEE Global Commun. Conf. (GLOBECOM), Austin, TX, Dec. 2014, pp. 1-6.

[6] Z. Ding, F. Adachi, and H. Poor, "The application of MIMO to non-orthogonal multiple access," IEEE Trans. Wireless Commun., vol. 15, no. 1, pp. 537-552, Jan. 2016.

[7] Z. Ding, M. Peng, and H. V. Poor, "Cooperative non-orthogonal multiple access in 5G systems," IEEE Commun. Lett., vol. 19, no. 8, pp. 1462-1465, Aug. 2015. 
[8] C. Zhong and Z. Zhang, "Non-orthogonal multiple access with cooperative full-duplex relaying," IEEE Commun. Lett., vol. PP, no. 99, pp. 1-1, Sep. 2016.

[9] A. Sendonaris, E. Erkip, and B. Aazhang, "User cooperation diversity - part I: system description," IEEE Trans. Commun., vol. 51, no. 11, pp. 1927-1938, Nov. 2003.

[10] J. N. Laneman, D. N. C. Tse, and G. W. Wornell, "Cooperative diversity in wireless networks: efficient protocols and outage behavior," IEEE Trans. Inf. Theory, vol. 50, no. 12, pp. 3062-3080, Dec. 2004.

[11] P. A. Anghel and M. Kaveh, "Exact symbol error probability of a cooperative network in a Rayleigh-fading environment," IEEE Trans. Wireless Commun., vol. 3, no. 5, pp. 1416-1421, Sep. 2004.

[12] M. O. Hasna and M.-S. Alouini, "A performance study of dual-hop transmissions with fixed gain relays," IEEE Trans. Wireless Commun., vol. 3, no. 6, pp. 1963-1968, Nov. 2004.

[13] — - "End-to-end performance of transmission systems with relays over Rayleigh-fading channels," IEEE Trans. Wireless Commun., vol. 2, no. 6, pp. 1126-1131, Nov. 2003.

[14] A. Bletsas, A. Khisti, D. P. Reed, and A. Lippman, "A simple cooperative diversity method based on network path selection," IEEE J. Sel. Areas Commun., vol. 24, no. 3, pp. 659-672, Mar. 2006.

[15] I. Krikidis, J. Thompson, S. Mclaughlin, and N. Goertz, "Amplify-and-forward with partial relay selection," IEEE Commun. Lett., vol. 12, no. 4, pp. 235-237, Apr 2008.

[16] D. B. da Costa and S. Aissa, "End-to-end performance of dual-hop semi-blind relaying systems with partial relay selection," IEEE Trans. Wireless Commun., vol. 8, no. 8, pp. 4306-4315, Aug 2009.

[17] H. Ding, J. Ge, and Z. Jiang, "Asymptotic performance analysis of amplify-and-forward with partial relay selection in Rician fading," Electronics Lett., vol. 46, no. 3, pp. 263-264, Feb. 2010.

[18] D. B. da Costa and S. Aissa, "Capacity analysis of cooperative systems with relay selection in Nakagami- $m$ fading," IEEE Commun. Lett., vol. 13, no. 9, pp. 637-639, Sep. 2009.

[19] Y. Liu, Z. Ding, M. Elkashlan, and H. V. Poor, "Cooperative non-orthogonal multiple access with simultaneous wireless information and power transfer," IEEE J. Sel. Areas Commun., vol. 34, no. 4, pp. 938-953, Apr. 2016.

[20] J.-B. Kim and I.-H. Lee, "Capacity analysis of cooperative relaying systems using non-orthogonal multiple access," IEEE Commun. Lett., vol. 19, no. 11, pp. 1949-1952, Nov. 2015.

[21] _ _ "Non-orthogonal multiple access in coordinated direct and relay transmission," IEEE Commun. Lett., vol. 19, no. 11, pp. 2037-2040, Nov. 2015.

[22] Z. Ding, H. Dai, and H. V. Poor, "Relay selection for cooperative NOMA," IEEE Commun. Lett., vol. 5, no. 4, pp. 416-419, Aug. 2016.

[23] J. Men and J. Ge, "Non-orthogonal multiple access for multiple-antenna relaying networks," IEEE Commun. Lett., vol. 19, no. 10, pp. 1686-1689, Oct. 2015.

[24] — - "Performance analysis of non-orthogonal multiple access in downlink cooperative network," IET Commun., vol. 9, no. 18, pp. 2267-2273, Dec. 2015.

[25] M. Abramowitz and I. A. Stegun, Handbook of Mathematical Functions with Formulas, Graphs, and Mathematical Tables. New York: Dover, 1972.

[26] I. S. Gradshteyn and I. M. Ryzhik, Table of integrals, series and products, 6th ed. San Diego, CA: Academic, 2000. 


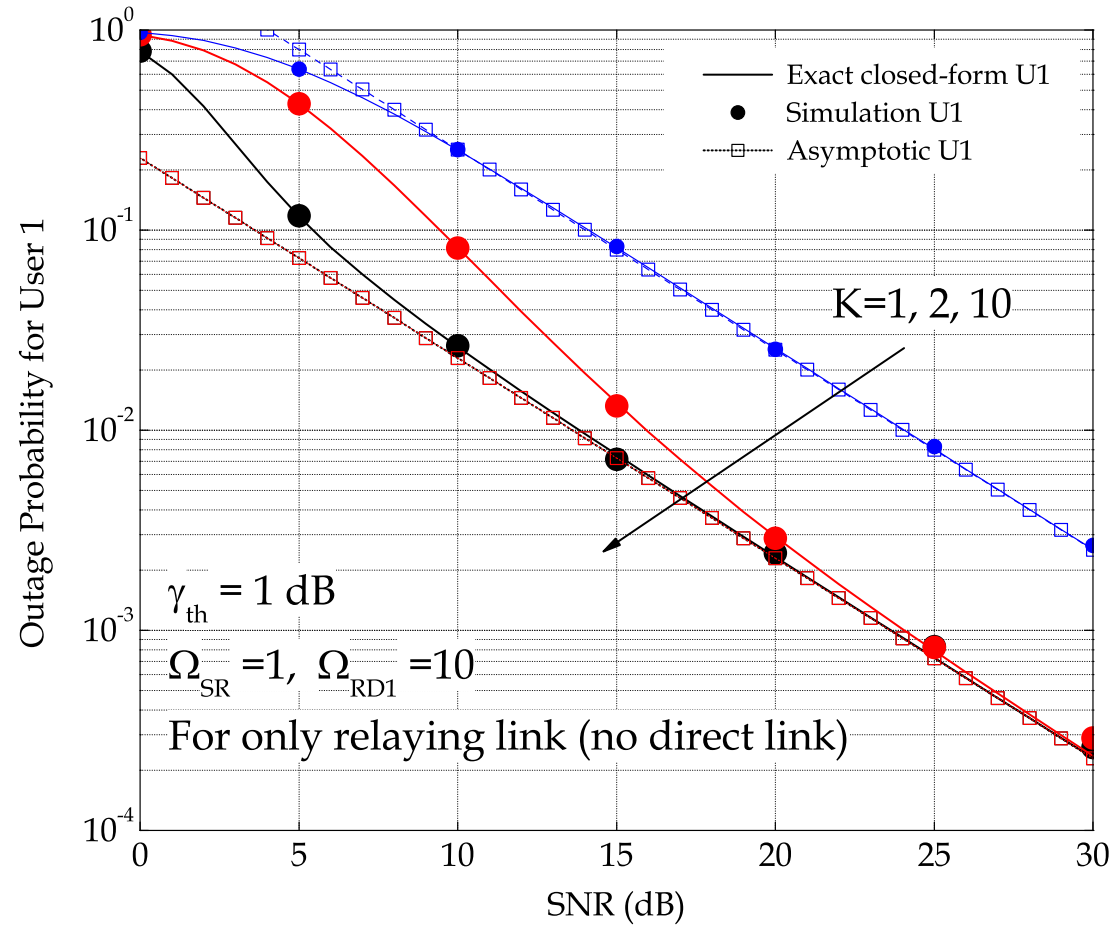

Fig. 2: OP of User 1 versus average SNR when number of relays $K=1,2,10$ with relaying link only. 


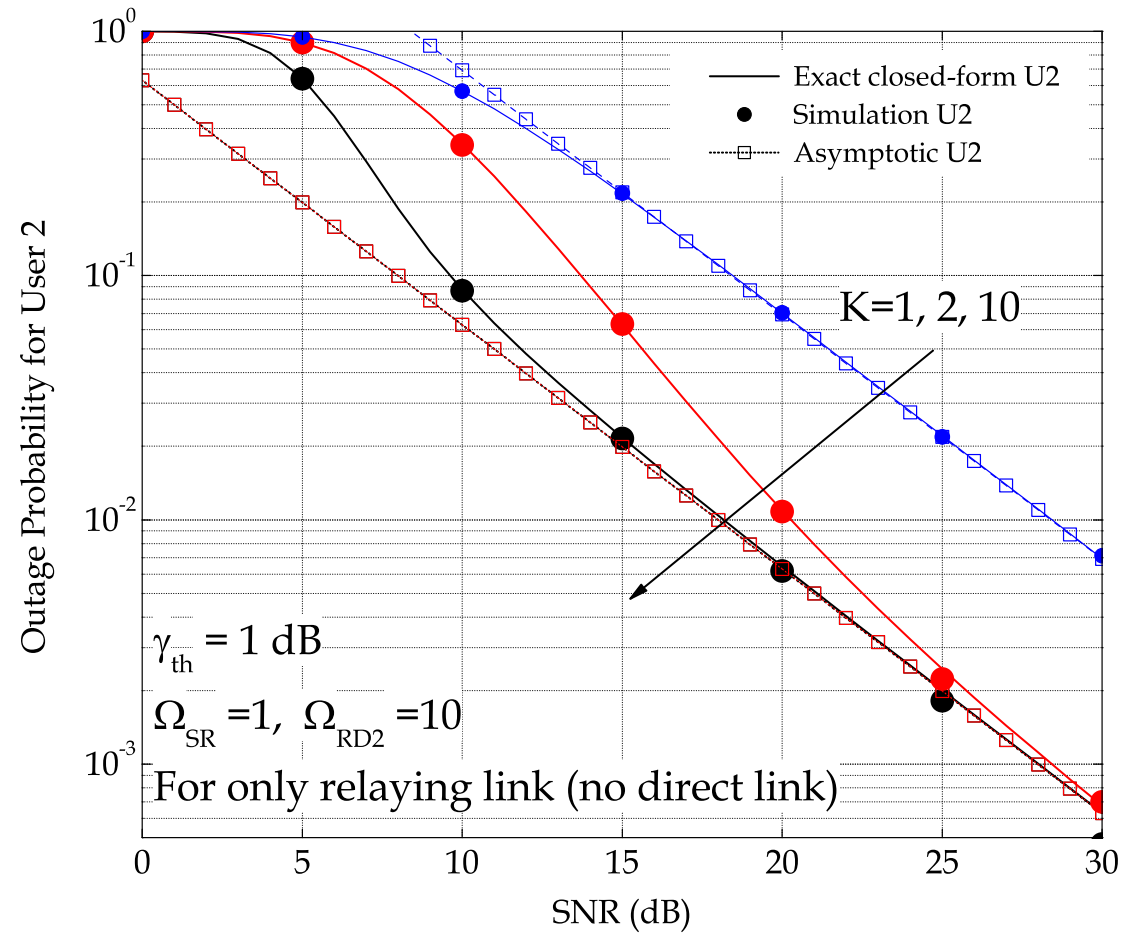

Fig. 3: OP of User 2 versus average SNR when number of relays $K=1,2,10$ with relaying link only.

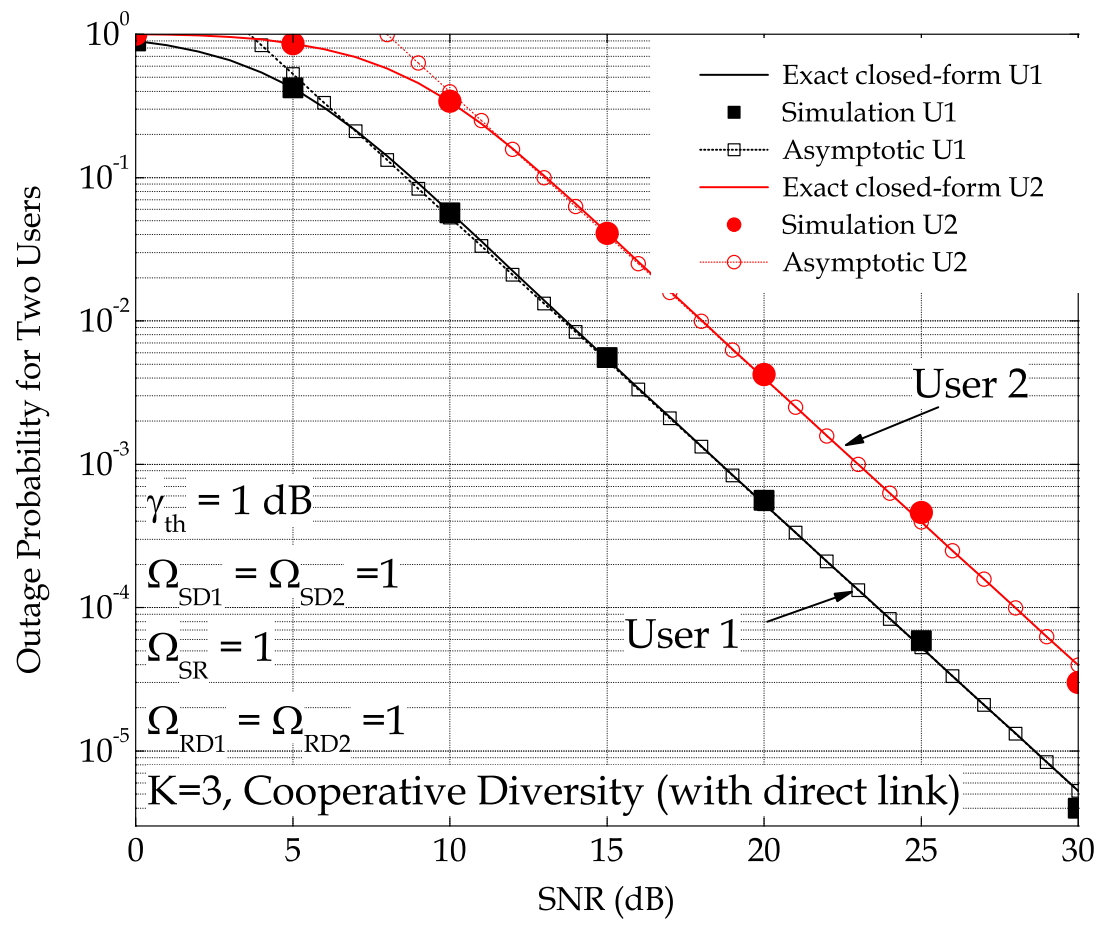

Fig. 4: OP of two users versus average SNR for $K=3$ with cooperative diversity. 


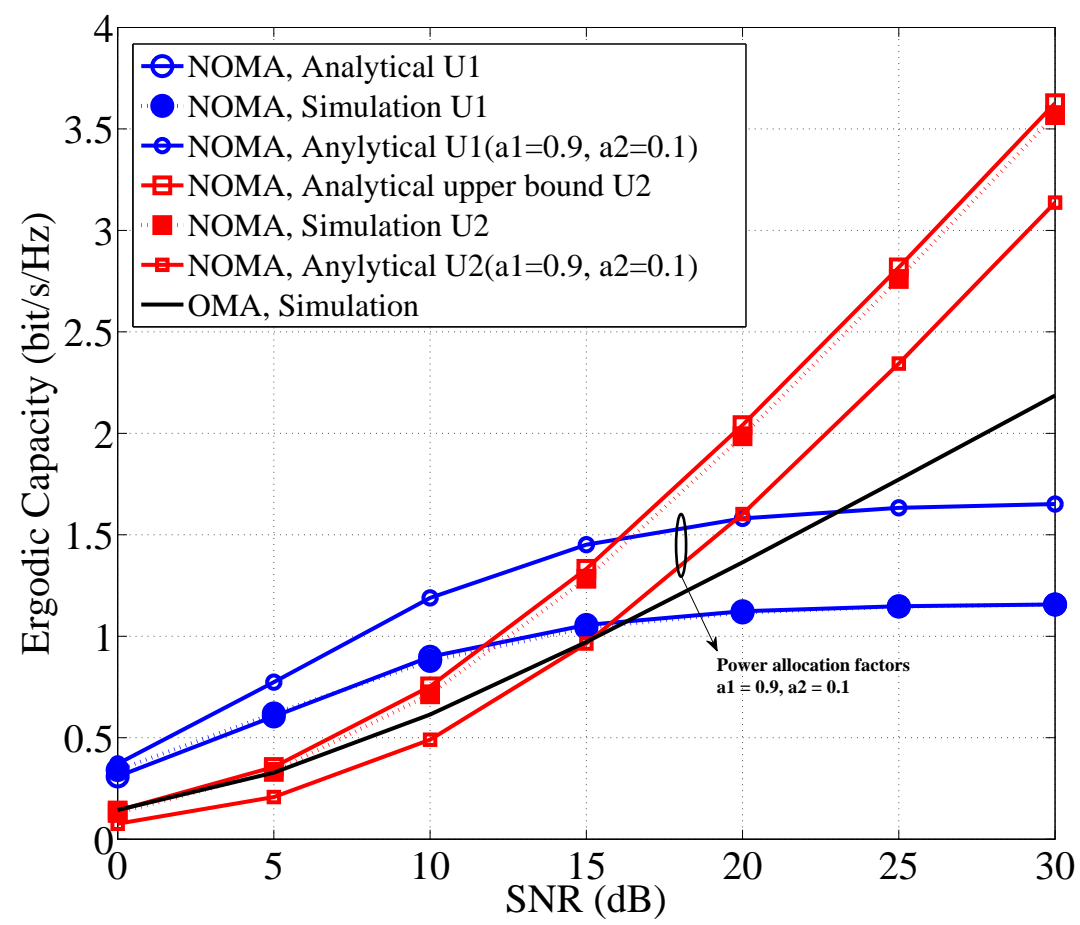

Fig. 5: Ergodic capacity of two users versus average SNR with cooperative diversity when $K=3$ and $\Omega_{\mathrm{SD}_{1}}=\Omega_{\mathrm{SD}_{2}}=\Omega_{\mathrm{SR}}=\Omega_{\mathrm{RD}_{1}}=\Omega_{\mathrm{RD}_{2}}=1$.

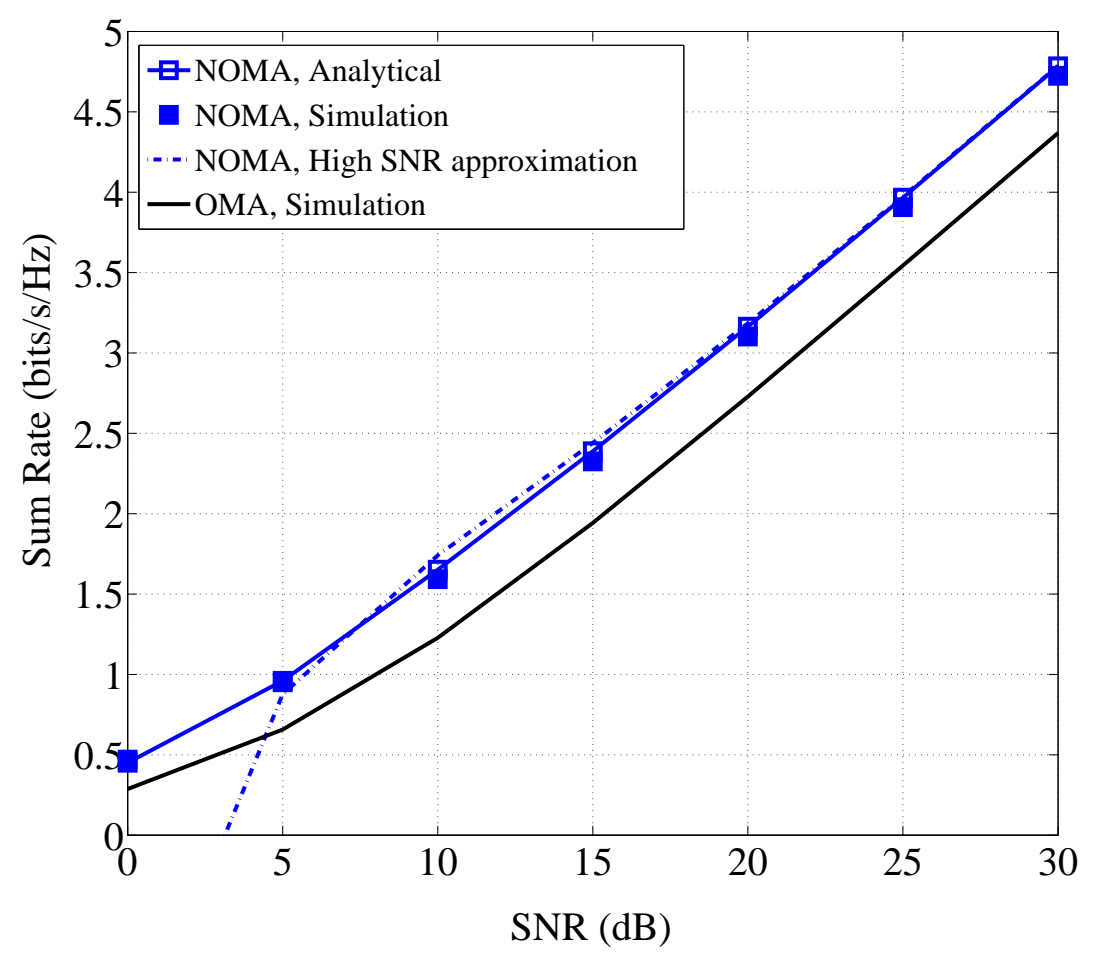

Fig. 6: Sum rate and its high SNR approximation versus average SNR when $K=3$ and $\Omega_{\mathrm{SD}_{1}}=\Omega_{\mathrm{SD}_{2}}=\Omega_{\mathrm{SR}}=\Omega_{\mathrm{RD}_{1}}=\Omega_{\mathrm{RD}_{2}}=1$. 